\title{
Photoluminescence Study of Bulk GaN Doped with Beryllium
}

\author{
M. JAWOREK ${ }^{a}$, A. WysmoŁeK ${ }^{a, *}$, M. KAmińskA ${ }^{a}$,
} A. Twardowski ${ }^{a}$, M. BoćKOWski $^{b}$ AND I. GrzeGORY ${ }^{b}$

${ }^{a}$ Institute of Experimental Physics, Warsaw University

Hoża 69, 00-681 Warsaw, Poland

${ }^{b}$ Institute of High Pressure Physics, Polish Academy of Sciences, Warsaw, Poland

\begin{abstract}
Photoluminescence of bulk GaN:Be grown by high pressure method is presented. The investigated crystals show well-resolved photoluminescence due to free and bound excitons similar to that observed for homoeptitaxial GaN layers. In addition to the excitonic transitions, pronounced luminescence band at $3.38 \mathrm{eV}$, due to Be acceptor, is observed. It was found that temperature behavior of this emission is typical of donor- and conduction band-acceptor transitions. The optical activation energy of Be acceptor is obtained to be of $60 \pm 15 \mathrm{meV}$.
\end{abstract}

PACS numbers: 78.55.Cr, 71.55.-i, 71.35.--y

\section{Introduction}

Efficient and controllable p-type doping is still important for GaN based optoelectronic devices, working in blue and ultraviolet range. In spite of the fact that Mg-doping is efficient enough to achieve mass production of blue lasers and LEDs, further improvement of the $p$-type GaN doping is desirable. One of the possible solutions of this problem would be to use beryllium, which, due to its large electronegativity and the absence of $d$-electrons, is predicted by ab initio calculations to be the shallowest acceptor in GaN with an ionization energy of $60 \mathrm{meV}[1]$.

In spite of the promising theoretical predictions, practical realization of this idea appeared to be very difficult. The main problem with the $\mathrm{Be}$ in $\mathrm{GaN}$ is that it easily incorporates on interstitial sites, where it acts as a donor $[2,3]$. Nevertheless it was shown that small concentrations of beryllium acting as acceptors can be introduced into epitaxial GaN layers by MBE method [4]. It has been found

${ }^{*}$ corresponding author; e-mail: Andrzej Wysmolek@fuw.edu.pl 
that in this case Be acceptor induces a characteristic luminescence band around $3.38 \mathrm{eV}[4,5]$.

In this paper we show that Be can be successfully introduced into bulk GaN grown using a high pressure method. A high optical quality of these crystals allowed detailed photoluminescence studies of Be-related luminescence.

\section{Samples and experiment}

Strain free GaN bulk crystals of wurtzite structure doped with Be and Mn were grown at High Pressure Research Center, from the solution of nitrogen in liquid gallium under 1.5 GPa pressure of $\mathrm{N}_{2}$ and at elevated temperatures of about $1500^{\circ} \mathrm{C}$. Doping with Be and Mn was obtained by adding these elements to the solution. Several samples with different nominal Be and Mn concentrations were studied.

Photoluminescence (PL) experiments were performed in a temperature range between 4.2 and $150 \mathrm{~K}$ using a continuous-flow cryostat. PL was excited by means of $3.813 \mathrm{eV}$ line of $\mathrm{He}-\mathrm{Cd}$ laser. The exciting light was focused on the sample using microscope objective providing spot size of about few microns. The excitation density resulting on the sample is estimated to be in the range of $10^{4} \mathrm{~W} / \mathrm{cm}^{2}$. The spectra were analyzed with a single $0.5 \mathrm{~m}$ monochromator equipped with a UV-enhanced CCD camera. The spectral resolution provided by the experimental setup was better than $0.2 \mathrm{meV}$.

\section{Results and discussion}

Low temperature photoluminescence spectra of the investigated samples show sharp emission lines due to neutral donor bound exciton $\left(\mathrm{D}^{0} \mathrm{X}\right)$ as well as neutral acceptor bound exciton $\left(\mathrm{A}^{0} \mathrm{X}\right)$ (see Fig. 1). At higher energies less intense structures due to free excitons $\mathrm{A}$ and $\mathrm{B}$ can be resolved. This is in contrast to usual luminescence of high pressure bulk GaN for which typically no structures of $\mathrm{PL}$ have been detected. The observed positions of exciton lines $\left(\mathrm{A}^{0} \mathrm{X}-3.465 \mathrm{eV}\right.$, $\mathrm{D}^{0} \mathrm{X}-3.469 \mathrm{eV}, \mathrm{X}_{\mathrm{A}}-3.477 \mathrm{eV}, \mathrm{X}_{\mathrm{B}}-3.483 \mathrm{eV}$ ) correspond exactly to those measured for unstrained homoepitaxial GaN layers [6]. The half-widths of $\mathrm{D}^{0} \mathrm{X}$ and $\mathrm{A}^{0} \mathrm{X}$ lines are of about $1 \mathrm{meV}$. These observations confirm a high optical quality of the investigated material and prove that this is truly strain free GaN.

In a low energy part of the spectrum, at $3.268 \mathrm{eV}$, donor-acceptor and/or conduction band acceptor luminescence $(\mathrm{D}-\mathrm{A} / \mathrm{e}-\mathrm{A})$, involving native defects are observed [7]. The most interesting luminescence, which we attribute to donoracceptor and/or conduction band-acceptor transition involving beryllium appears at energies close to $3.38 \mathrm{eV}$. This energy corresponds very well to the emission previously identified as due to Be acceptor [4]. As it is seen in Fig. 2, relative emission intensity and energy of this line depend slightly on the position on the 


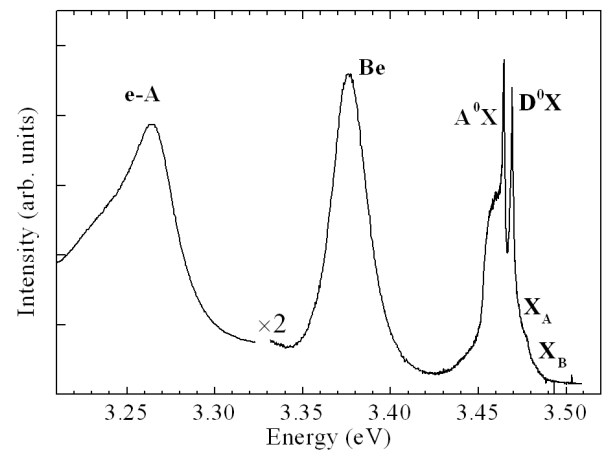

Fig. 1. $\quad \mu$-PL spectrum of GaN:Be, Mn taken at liquid helium temperature.

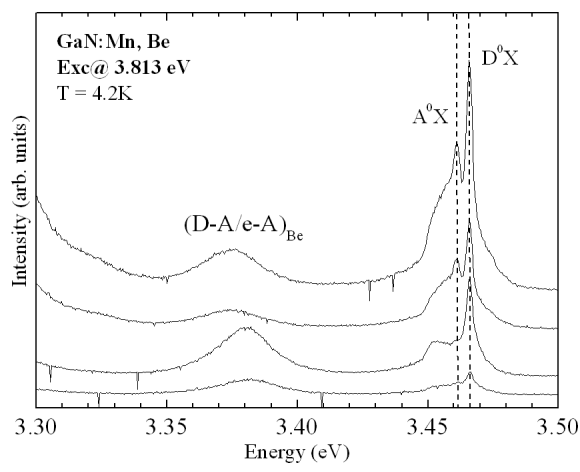

Fig. 2. The spectra measured at different positions of the GaN:Be sample.

sample. Since the position of excitonic transitions remains unchanged, the observed energy fluctuations $(\sim 10 \mathrm{meV})$ cannot be explained by inhomogeneous strain distribution, and result rather from the fluctuations of Be concentration.

In order to confirm our identification of the $3.38 \mathrm{eV}$ band as due to donor-Beacceptor and/or conduction-band Be acceptor (D-A/e-A) transitions, its temperature behavior was measured and compared with the behavior of typical $\mathrm{D}-\mathrm{A} / \mathrm{e}-\mathrm{A}$ band observed at $3.268 \mathrm{eV}$. The photoluminescence spectra measured in the temperature range between 4.2 and $145 \mathrm{~K}$ are presented in Fig. 3. At first sight, both $3.268 \mathrm{eV}$ and $3.38 \mathrm{eV}$ bands show very similar temperature characteristics. Their energy positions (Fig. 4) move in a very similar way towards higher energies with increasing temperature. In spite of the fact that at $4.2 \mathrm{~K}$ the FWHM of the $3.268 \mathrm{eV}$ band $(15 \mathrm{meV})$ is much larger than that of $3.38 \mathrm{eV}$ band $(6.5 \mathrm{meV})$, their temperature changes are almost the same (Fig. 5). The above behavior confirms donor-acceptor and/or conduction band-acceptor type transitions of the both $3.268 \mathrm{eV}$ and $3.38 \mathrm{eV}$ bands. As can be seen in Fig. 5, the observed energy shift is not linear and does not follow $(1 / 2) \mathrm{kT}$ slope, which is expected for free electrons recombining with the neutral acceptors. This could result from modifica- 


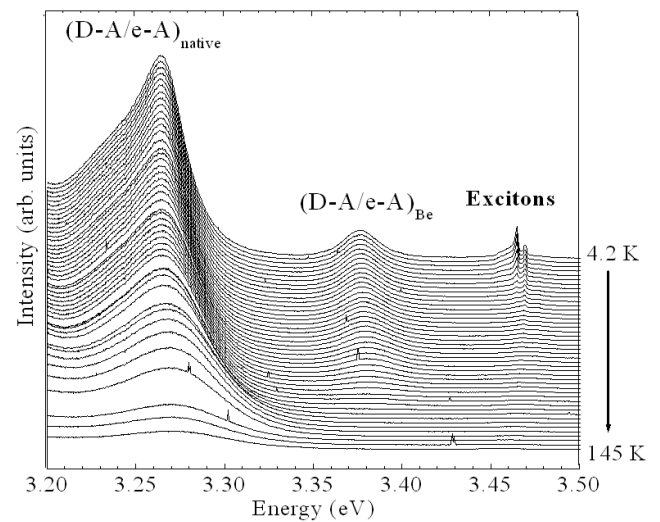

Fig. 3. Photoluminescence spectra of GaN:Mn, Be at various temperatures and the temperature behavior of $\mathrm{D}-\mathrm{A}_{\mathrm{Be}}$ intensity.

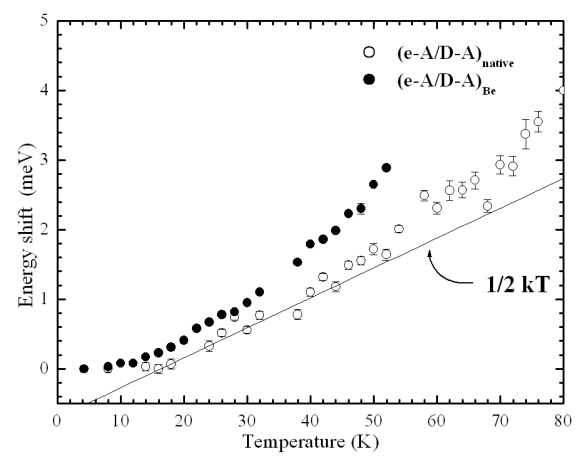

Fig. 4. The temperature dependence of the emission energy corresponding to $(\mathrm{D}-\mathrm{A} / \mathrm{e}-\mathrm{A})_{\mathrm{Be}}\left(\right.$ closed circles) and $(\mathrm{D}-\mathrm{A} / \mathrm{e}-\mathrm{A})_{\text {native }}$ band (open symbols).

tion of the density of states in the conduction band cased by potential fluctuations, which result from the compensation of the material.

In order to evaluate activation energy $E_{\mathrm{A}}$ corresponding to the Be acceptor, the temperature dependence of the luminescence intensity was fitted by a standard formula:

$$
I(T)=I_{0} /\left[1+A \exp \left(-E_{\mathrm{A}} / k T\right)\right]
$$

where $I(T)$ - integrated emission intensity at temperature $T, A$ - a fitting parameter.

As shown in Fig. 6, the formula (1) fits experimental data quite well and provides activation energy of the native acceptor and $\mathrm{Be}$ acceptor, equal to $E_{\mathrm{A}}=(165 \pm 10) \mathrm{meV}$ and $(60 \pm 15) \mathrm{meV}$, respectively. The value obtained for Be acceptor remains in good agreement with the theoretical predictions [3] and is slightly smaller than that obtained from the investigations of MBE grown material [4]. 


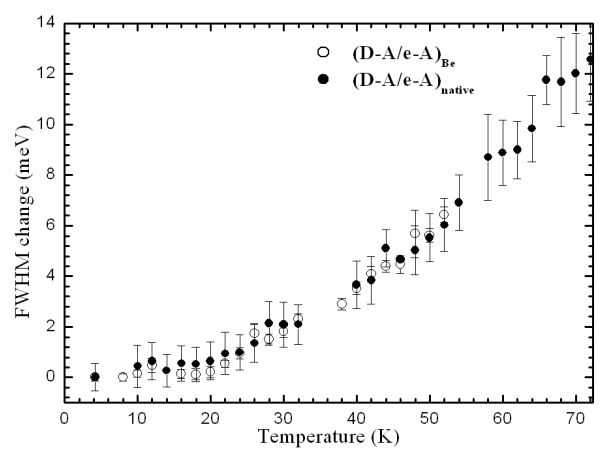

Fig. 5. The temperature dependence of the FWHM of (D-A/e-A) Be (open symbols) and $(\mathrm{D}-\mathrm{A} / \mathrm{e}-\mathrm{A})_{\text {native }}$ lines. The FWHM of these lines are $6.5 \mathrm{meV}$ and $15 \mathrm{meV}$, respectively.

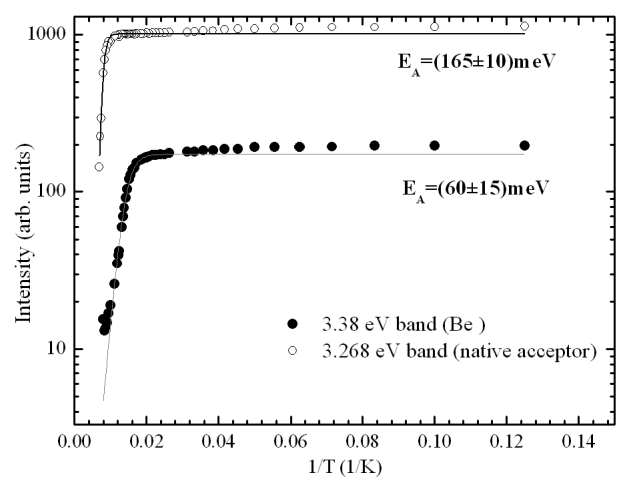

Fig. 6. The temperature dependence of the intensity of $3.38 \mathrm{eV}$ (closed symbols) band related to Be acceptor and $3.268 \mathrm{eV}$ corresponding to the native acceptor (open symbols).

The obtained result could be compared with the estimation of acceptor ionization energy based on the observed emission energy corresponding to the donoracceptor or conduction band - acceptor transitions. Since it is very difficult to separate the contribution from both of the processes, two possibilities should be taken into account. In the case, when e-A transition dominates the recombination processes, the energy difference between band gap and the energy of the maximum of the $\mathrm{e}-\mathrm{A}$ emission is a direct measure of the acceptor ionization. Taking into account that for unstrained $\mathrm{GaN}, E_{\mathrm{g}}=3.504 \mathrm{eV}$ [8] the resulting difference, corresponding to the acceptor binding energy is estimated to be of $125 \mathrm{meV}$. In the case when the recombination is dominated by $\mathrm{D}-\mathrm{A}$ pair process, the acceptor binding energy can be described by the following formula:

$$
E_{\mathrm{A}}=E_{\mathrm{g}}-E_{\mathrm{D}}-E_{\mathrm{DAP}}+E_{\mathrm{C}}
$$

where $E_{\mathrm{D}}$ and $E_{\mathrm{C}}$ are the donor binding energy and the effective Coulomb interaction between bound electron and hole, respectively. 
Taking into account that the binding energy of a shallow donor in GaN is around $30-33 \mathrm{meV}[9]$ and effective Coulomb interaction could be estimated to be in the order of $60 \mathrm{meV}$ [4], the beryllium ionization resulting from the formula (2) is of about $92-95 \mathrm{meV}$. Since usually optical transition energies are bigger than thermal activation energies of the acceptor centers, the obtained estimation of the Be ionization energy, being in the range of $90-125 \mathrm{meV}$, seems to be consistent with the results of the temperature dependent luminescence studies.

\section{Conclusions}

The investigated GaN:Be, Mn bulk crystals show a very good optical quality comparable with that of homoepitaxial layers, enabling the observation of narrow emission lines due to recombination of bound excitons, as well as of pronounced emission band due to beryllium acceptor observed at $3.38 \mathrm{eV}$. The optical activation energy of Be center is obtained to be of $60 \pm 15 \mathrm{meV}$ and is consistent with the theoretical predictions.

\section{Acknowledgments}

This work was partially supported by the State Committee for Scientific Research grant PBZ-KBN-044/P03/2001.

\section{References}

[1] F. Bernardini, V. Fiorentini, A. Bosin, Appl. Phys. Lett. 70, 2990 (1997).

[2] H.T. Wang, L.S. Tan, E.F. Chor, J. Cryst. Growth 268, 489 (2004).

[3] J. Neugebauer, C.G. Van der Walle, Appl. Phys. Lett. 85, 3003 (1999).

[4] H. Teisseyre, I. Gorczyca, N.E. Christensen, A. Svane, F.B. Naranjo, E. Calleja, J. Appl. Phys. 97, 043704 (2005).

[5] E. Calleja, M.A. Sanchez-Garcia, F. Calle, F.B. Naranjo, E. Munoz, U. Jahn, K. Ploog, J. Sanchez, J.M. Calleja, K. Saarinen, H. Hautojarvi, Mater. Sci. Eng. $B$ 82, 2 (2001).

[6] K. Pakula, A. Wysmolek, K.P. Korona, J.M. Baranowski, R. Stepniewski, I. Grzegory, M. Bockowski, J. Jun, S. Krukowski, M. Wroblewski, S. Porowski, Solid State Commun. 97, 919 (1996).

[7] O. Lagerstedt, B. Monemar, J. Appl. Phys. 45, 2266 (1974).

[8] K.P. Korona, A. Wysmołek, K. Pakuła, R. Stȩpniewski, J.M. Baranowski, I. Grzegory, B. Łucznik, M. Wróblewski, S. Porowski, Appl. Phys. Lett. 69, 788 (1996).

[9] R. Stȩpniewski, A. Wysmołek, M. Potemski, Phys. Status Solidi A 201, 181 (2004) and references therein. 